\title{
Editorial
}

\section{Investing in clinical health promotion}

\section{Hanne Tønnesen}

\section{About the AUTHOR}

Editor-in-Chief
Director, WHO-CC, Clinical
Health Promotion Centre,
Bispebjerg \& Frederiks-
berg Hospital, University of
Copenhagen, Copenhagen,
Denmark.
Professor at Lund University,
Skåne University Hospital,
Malmö, Sweden
Professor, University of
Southern Denmark
CEO, International HPH
Secretariat
President, Clinical Health
Promotion Society

Contact: Hanne Tønnesen Hanne.Tonnesen@regionh.dk

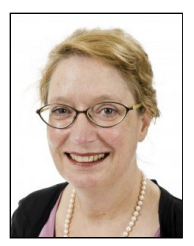

Investments in health are returned within weeks for patient-centered health promotion and over years for populationbased health promotion.

More and more evidence indicates that investing in clinical health promotion improves the outcome significantly for patients treated in hospitals and other health services. The better outcomes are seen immediately on short term - within weeks, actually. Furthermore, these short-term outcomes are followed by a clear long-term effect measurable in public health (1-5).

\section{Health of the population}

There are many good examples showing that public health investment is cost-effective over time. According to the recent WHO publication The case for investing in public health, focal points for action are especially the tobacco control, limitation of alcohol intake, healthy nutrition, and physical activity (6). By doing nothing, the costs are tremendous and so are the loss of years with healthy life (defined as 1 DALY $=1$ Disability-Adjusted Life Year). The WHO publication is praiseworthy and easy to use. It targets the economic calculations of disabilities directly related to lifestyle.

In the best cases, the financial outcome of an investment in health is multiplied within a few years, which is a short time from an economic perspective. In contrast, in the worst cases, the costs of not acting are tremendous. Thus, the lost years of healthy life for the European Region are 17.7 million DALYs for tobacco and 17.3 million DALYs for harmful alcohol use. An unhealthy diet is responsible for 15.3 million DALYs and physical inactivity for 8.2 million DALYs. There is no reason to believe that lost healthy years should be much better in other regions, so the impact of unhealthy lifestyles is extreme - both for the individuals experiencing the disabilities, for the health care services, and for the societies at large.

In comparison, the road traffic injuries are responsible for 3.6 million DALYs and the environmental risk for 2.5 million DALYs, which include occupational risk, air pollution, unsafe water and sanitation, as well as global climate changes amongst others (6). The important publication from WHO include and recommendations on how to strengthen public health services as well as preventive procedures. The effects for individuals might be minor, but the total effect for the total population is measurable within few years and remarkable in the longer term (6).

\section{Health of the patient}

The health of the patient and the needs for health promotion add additional perspectives (see figure 1). In contrast to the population-level, almost all patients are entering fixed treatment programs for their individual illness, leaving almost no time to wait for public health interventions. They need especially effective

Figure 1: Improvement of Health of the population and patients' health (dotted line) and effect of intervention

Unhealthy lifestyle

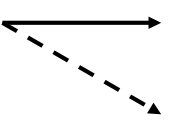

Better lifestyle
Lifestyle-related illness and problems

Aggravation of treatment outcome of diseases

Reduced lifestyle-related illness and problems

Improved treatment outcome of diseases 
health promotion programs that start immediately at their first contact to the hospital or another health service, so that they can benefit from better treatment results here and now. The duration of their treatment is counted in days and the recovery period in weeks.

Unfortunately, most reports on health economy and lifestyles lack the additional patient perspective and the costs from the aggravating effects that poor lifestyles have on treatments and prognoses of illnesses, not lifestyle-related illnesses (6). These effects include the additional complications after surgery developed by smokers and harmful drinkers, the knee prosthetic replacements indicated by obesity, the aggravation of diabetes, asthma, and communicable diseases, the high pre-mortality in psychiatric patients, and the heavy influence on mother and child during and after pregnancy among many others (7).

Tremendous and unnecessary suffering and costs arise from second surgeries, re-admissions and prolonged recoveries caused by the smoking, hazardous drinking, malnutrition, overweight, and physical inactivity of patients with poor lifestyles, who are being treated for illnesses not originating from their lifestyles. Thus, the burden of disease becomes unnecessarily heavy because the additional burden is in fact preventable by adding clinical health promotion to all patient pathways for smokers and hazardous drinkers, as well as for malnourished, overweight, and physical inactive patients.

A full-scaled calculation of costs would reveal the best possible actions for patients and the worst-case cost scenario of not acting, allowing national and local health services to choose the best interventions in each sector. There is no doubt that the majority of the health promotion should take place within families and at workplaces, schools, and institutions outside of the hospitals and other health services. However, the large majority of patients have special needs for immediate, effective, and fast-track health promotion, which has shown to be very cost-effective in the very short term (8).

Only effective clinical health promotion improves patients' treatment results

About $80-90 \%$ of patients in hospitals have one or more of the five lifestyles that reduce their treatment outcome: smoking, hazardous drinking, malnutrition, overweight and physical inactivity (8). Even the most competent specialist clinician and general practitioner, the most talented management, or the most skilled nurse, midwife, and physiotherapist cannot make the lifestyle-related complications disappear by neglecting them. There is only one way to improve the treatment outcome. It is therefore necessary to integrate patient-centered health promotion in hospitals and health services in line with the other interventions, such as medication and surgery.

However, in order to harvest the benefits of clinical health promotion, cost-effective lifestyle intervention programs with a very low number needed to treat (NNT) are required in addition to significance, such as the gold standard program (GSP) for smoking cessation interventions, working well across ages, settings, and social gradients (9).

If you use the GSP as in the first randomized intervention study on smokers scheduled for hip and knee replacement therapy (10), NNT is 1-2 to get one extra successful quitter and 3-4 to get one extra patient free of complications after this kind of elective surgery.

If instead you choose a brief intervention program, you will get an NNT at 20 per successful quitter without any effect on the complications at all (1).

\section{Dos and don'ts}

When you want to improve the treatment results for patients with poor lifestyles, be aware that only the most intensive health promotion programs have an immediate effect on both lifestyle and the treatment results. Today, most patients receive very fast-track pathways, leaving no time for useless health promotion interventions. Furthermore, there is no clinical, scientific, or economic rationale for delivering ineffective programs to our patients. They are already carrying a heavy burden of diseases and injuries.

\section{References}

(1) Thomsen T, Villebro N, Møller AM. Interventions for preoperative smoking cessation. Cochrane Database Syst Rev. 2014 Mar 27;3:CD002294.

(2) Oppedal K, Møller AM, Pedersen B, Tønnesen H. Preoperative alcohol cessation prior to elective surgery. Cochrane Database Syst Rev.2012 Jul 11;7:CD008343

(3) Nielsen PR, Jørgensen LD, Dahl B, Pedersen T, Tønnesen H. Prehabilitation and early rehabilitation after spinal surgery: randomized clinical trial. Clin Rehabil 2010; 24:137-48.

(4) Burden S, Todd C, Hill J, Lal S. Pre-operative nutrition support in patients undergoing gastrointestinal surgery Cochrane Database Syst Rev. 2012 Nov 14;11:CD008879.

(5) Taylor G, McNeill A, Girling A, Farley A, Lindson-Hawley N, Aveyard P (2014). Change in mental health after smoking cessation: systematic review and metaanalysis. BMJ 2014; 348:g1151

(6) World Health Organization: The case for investing in public health - A Public health summary report for EPHO 8. WHO Regional Office Europe, UN City Copenhagen. 2014

(7) Tønnesen H (Ed). Engage in the Process of Change; Facts and Methods: World Health Organization 2012, Copenhagen.

(8) Oppedal $\mathrm{K}$ et al. Health and the need for health promotion in hospital patients. Eur J Publ Health 2011; 21:744-9

(9) Kehlet M et al. The Gold Standard Program for Smoking Cessation is Effective for Participants Over 60 Years of Age. Int. J. Environ. Res. Public Health 2015; 12, 2574-2587; doi:10.3390/ijerph120302574

(10) Møller A et al. Effect of preoperative smoking intervention on postoperative complications: a randomised clinical trial. Lancet 2002; 359:114-117; doi: http://dx.doi.org/10.1016/S0140-6736(02)07369-5 